\title{
Probing dynamics of micro-magnets with multi-mode superconducting resonator
}

I. A. Golovchanskiy, N. N. Abramov, V. S. Stolyarov, I. V. Shchetinin, P. S. Dzhumaev, A. S. Averkin, S. N. Kozlov, A. A. Golubov, V. V. Ryazanov, and A. V. Ustinov

Citation: Journal of Applied Physics 123, 173904 (2018); doi: 10.1063/1.5025028

View online: https://doi.org/10.1063/1.5025028

View Table of Contents: http://aip.scitation.org/toc/jap/123/17

Published by the American Institute of Physics

\section{Articles you may be interested in}

Multi-directional emission and detection of spin waves propagating in yttrium iron garnet with wavelengths down to about $100 \mathrm{~nm}$

Applied Physics Letters 112, 192410 (2018); 10.1063/1.5026060

Double Fe-impurity charge state in the topological insulator $\mathrm{Bi}_{2} \mathrm{Se}_{3}$

Applied Physics Letters 111, 251601 (2017); 10.1063/1.5002567

Phase control of spin waves based on a magnetic defect in a one-dimensional magnonic crystal Applied Physics Letters 112, 142405 (2018); 10.1063/1.5024541

Enhanced interfacial Dzyaloshinskii-Moriya interaction and isolated skyrmions in the inversion-symmetry-broken $\mathrm{Ru} / \mathrm{Co} / \mathrm{W} / \mathrm{Ru}$ films

Applied Physics Letters 112, 192406 (2018); 10.1063/1.5029857

Amplification and stabilization of large-amplitude propagating spin waves by parametric pumping Applied Physics Letters 112, 042402 (2018); 10.1063/1.5019357

Spin wave steering in three-dimensional magnonic networks

Applied Physics Letters 112, 122404 (2018); 10.1063/1.5023138

\section{PHYSICS TODAY}

MANAGER'S GUIDE WHITEPAPERS
Accelerate R\&D with Multiphysics Simulation

\section{READ NOW}

PRESENTED BY

マ๐OMSOL 


\title{
Probing dynamics of micro-magnets with multi-mode superconducting resonator
}

\author{
I. A. Golovchanskiy, ${ }^{1,2}$ N. N. Abramov, ${ }^{2}$ V. S. Stolyarov, ${ }^{1,3,4,5}$ I. V. Shchetinin, ${ }^{2}$ \\ P. S. Dzhumaev, ${ }^{6}$ A. S. Averkin, ${ }^{2}$ S. N. Kozlov, ${ }^{1,3,5}$ A. A. Golubov, ${ }^{1,7}$ V. V. Ryazanov, ${ }^{2,3,4}$ \\ and A. V. Ustinov ${ }^{2,8}$ \\ ${ }^{1}$ Moscow Institute of Physics and Technology, State University, 9 Institutskiy per., Dolgoprudny, \\ Moscow Region 141700, Russia \\ ${ }^{2}$ National University of Science and Technology MISIS, 4 Leninsky Prosp., Moscow 119049, Russia \\ ${ }^{3}$ Institute of Solid State Physics (ISSP RAS), Chernogolovka, 142432 Moscow Region, Russia \\ ${ }^{4}$ Solid State Physics Department, Kazan Federal University, 420008 Kazan, Russia \\ ${ }^{5}$ Fundamental Physical and Chemical Engineering Department, MSU, 119991 Moscow, Russia \\ ${ }^{6}$ National Research Nuclear University MEPhI, 31 Kashirskoye sh., 115409 Moscow, Russia \\ ${ }^{7}$ Faculty of Science and Technology and MESA + Institute for Nanotechnology, University of Twente, \\ 7500 AE Enschede, The Netherlands \\ ${ }^{8}$ Physikalisches Institut, Karlsruhe Institute of Technology, 76131 Karlsruhe, Germany
}

(Received 6 February 2018; accepted 18 April 2018; published online 7 May 2018)

\begin{abstract}
In this work, we propose and explore a sensitive technique for investigation of ferromagnetic resonance and corresponding magnetic properties of individual micro-scaled and/or weak ferromagnetic samples. The technique is based on coupling the investigated sample to a high-Q transmission line superconducting resonator, where the response of the sample is studied at eigen frequencies of the resonator. The high quality factor of the resonator enables sensitive detection of weak absorption losses at multiple frequencies of the ferromagnetic resonance. Studying the microwave response of individual micro-scaled permalloy rectangles, we have confirmed the superiority of fluxometric demagnetizing factor over the commonly accepted magnetometric one and have depicted the demagnetization of the sample, as well as magnetostatic standing wave resonance. Published by AIP Publishing. https://doi.org/10.1063/1.5025028
\end{abstract}

\section{INTRODUCTION}

Individual micro- and sub-micro-scaled patterned ferromagnetic thin films are of interest for applications in magnetoelectronics, ${ }^{1-4}$ including various superconductor/ferromagnet (FM) hybrid structures. ${ }^{5-12}$ Characterization of properties of these ferromagnetic structures is imperative. Ferromagnetic resonance (FMR) study, and FMR absorption measurement with a vector network analyzer (VNA), in particular, ${ }^{13-15}$ is a very convenient tool for investigation of static and dynamic properties of ferromagnets (FMs). Indeed, a single resonance curve, i.e., the dependence of the FMR frequency on applied magnetic field, provides the saturation magnetization and effective magnetic anisotropy of extrinsic (shape anisotropy) or intrinsic (magneto-crystalline anisotropy) origin, according to the Kittel formulas. ${ }^{16,17}$ FMR frequency bandwidth provides the Gilbert damping parameter. ${ }^{15,18}$ Once an exchange spin wave activity is excited, it completes the list of basic magnetic properties with the exchange stiffness constant and the surface anisotropy (see, for example, Refs. 19-22).

However, detection of the FMR of individual small micro- or sub-micro-scaled FM can be challenging, mainly due to a small number of spins in a system and weak resonance response, and can be performed in two basic approaches. The first common approach implies investigation of the FMR response of infinite uniform FM thin film, derivation of its basic properties (i.e., the saturation magnetization $M_{s}$ and the anisotropy field $H_{a}$ ), and subsequent simulation of the dynamical response of the required size FM by means of micromagnetic simulation, ${ }^{23,24}$ employing experimentally defined properties. The second common approach implies FMR investigation of a large array of the required size FM samples ${ }^{25-34}$ taking into account magnetostatic interaction between the array elements or avoiding it. In this case, use of the array increases a number of spins in a system and ensures a measurable FMR response. Thus, none of basic approaches actually consider individual small FM samples.

The resonant response of an individual micro-scaled FM object can be studied effectively with several more sophisticated techniques. In particular, on the micrometer-scale the spin wave activity can be visualized with high sensitivity and spatial resolution using a combined setup, where the excitation of magnetization dynamics is done with microwave (MW) antennas, while visualization is performed using Brillouin light scattering microscopy ${ }^{35-38}$ or magnetic force microscopy. ${ }^{39-41}$ Among purely microwave experimental techniques, an impressive sensitivity for resonant spectroscopy of individual micro-scaled FMs has been achieved with microwave interferometers ${ }^{42}$ planar microwave micro-devices, ${ }^{43}$ and planar micro-resonators (PMRs). ${ }^{44,45}$ In case of planar micro-devices, an efficient coupling to small FMs is ensured by the localized inductance.

Recently, a fundamentally new development started to gain interest where the magnetic moment of individual small FM sample is excited by current oscillations in a hybrid Josephson junction. ${ }^{46-48}$ In this development, the FMR manifests itself as additional feature on the Josephson currentvoltage characteristics. However, FMR investigation with 
Josephson structures is technologically complex, nonversatile with respect to choice of the FM, and requires a detailed examination of Josephson properties prior to actual analysis of the FM response.

In this work, as an alternative, we propose to employ a high quality factor (high-Q) multi-mode transmission line superconducting resonator $^{49}$ for probing magnetization dynamics of individual micro-scaled ferromagnetic samples by means of the VNA-FMR measurement technique. ${ }^{14,15}$ When FM sample is placed near the current antinode of a standing wave inside the resonator, it couples efficiently to its eigen modes exciting the magnetization precession. Small changes in complex susceptibility of the sample, which are caused by magnetization dynamics effects, are directly related to the change of the resonator Q-factor ${ }^{50}$ and, thus, allow to depict small absorption losses induced by the FMR. The broadband capabilities of a conventional transmission line in a VNA-FMR measurements are replaced by multiple resonance modes, allowing to restore the entire dependence of the FMR frequency on applied magnetic field. The approach that we have implemented in this work is largely inspired by experiments on hybridisation of magnons and microwave photons within quantum circuits (see, for example, Refs. 51-53).

\section{EXPERIMENTAL DETAILS}

A schematic illustration of the investigated test chip is shown in Fig. 1(a). The test chip is based on the superconducting niobium $\lambda / 2$ coplanar wave guide (CPW) through type resonator ${ }^{49}$ with inductive coupling. The inductive coupling element consists of two overlapping $50 \Omega \mathrm{CPW}$ sections of different width, as shown in Figs. 1(a) and 1(b). In our experiment, we used a CPW with $17 \mu \mathrm{m}$ gap and $31 \mu \mathrm{m}$ central stripe width as the resonator, whereas coupled lines had $50 \mu \mathrm{m}$ gap and $87 \mu \mathrm{m}$ central stripe width, and the coupler section length was $110 \mu \mathrm{m}$ [Fig. 1(b)]. The total length $\approx 80 \mathrm{~mm}$ of the resonator on silicon substrate yields the fundamental resonance frequency $f_{0}=740 \mathrm{MHz}$. The coupling was numerically optimized using High Frequency Structure Simulator (HFSS) by adjusting coupler length to obtain coupling quality factor $Q_{c}=10^{4}$ at $f_{0}$. The resonator was folded into a meander. A single micro-scaled FM sample is placed at one end of the resonator, in close vicinity to the inductive coupler [see Fig. 1(a)], i.e., in the location of global current anti-node for all modes of the resonator, where the microwave (MW) magnetic fields are maximized. The central stripe was also narrowed down to $5 \mu \mathrm{m}$ under the FM sample. These means maximize the inductive coupling of the FM sample to the resonator. A $30 \mathrm{~nm} \mathrm{AlO}_{x}$ insulating layer is deposited between the superconducting and the ferromagnetic layers to avoid the superconducting proximity effect. In the absence of the insulating layer, the inverse proximity effect may suppress superconductivity at the constriction under the ferromagnetic sample in $\mathrm{Nb}$ and switch it into the resistive state, which would enable internal losses in the resonator and reduce the Q-factor. In experiments bias, magnetic field [green arrow in Fig. 1(a)] is oriented in-plane and parallel (PA) to the direction of the MW propagation, i.e., perpendicular $(\mathrm{PE})$ to the alternating magnetic field. In this work, the same experimental setup is used as in Ref. 33; all measurements were performed at $T=4 \mathrm{~K}$ and the magnetic field was scanned from 1500 Oe to -1500 Oe.

Two permalloy (Py) FM samples of $50 \times 7 \times 0.55 \mu \mathrm{m}^{3}$ size were investigated, with the longest side aligned along [Fig. 1(c)] and across [Fig. 1(d)] the transmission line. A comparative study of the MW responses of these samples allows to identify explicitly an impact of the shape anisotropy. Below, we refer to the experiment with FM sample aligned along the transmission line as PA (parallel) and to the one aligned across the transmission line as PE (perpendicular). Note, in both PA and PE geometries, FM sample is wider than the $\mathrm{Nb}$ line, which leads to highly inhomogeneous distribution of excitation MW fields across FM along $y$-axis [Fig. 1(a)], with the maximum amplitude focused in vicinity to the edges of the $\mathrm{Nb}$ line. ${ }^{14}$ In general, maximization of excitation fields enhances the coupling of the FM to the resonator, but inhomogeneity of excitation fields needs to be accounted if a study of spin waves is considered with the wavelength comparable to or smaller than the width of the $\mathrm{Nb}$ line.

The superconducting resonators were fabricated on $\mathrm{Si}$ substrate out of magnetron sputtered $100 \mathrm{~nm} \mathrm{Nb}$ film with superconducting critical temperature $T_{c} \simeq 9.02 \mathrm{~K}$ using optical lithography and plasma-chemical etching in $\mathrm{CF}_{4}+\mathrm{O}_{2}$ plasma. The base pressure in the growth chamber prior deposition was $5 \times 10^{-9}$ mbar. Prior to $\mathrm{Nb}$ deposition, the substrate was plasma-cleaned at $P_{A r}=2 \times 10^{-2}$ mbar, $60 \mathrm{~W} \mathrm{RF}$ power, and $500 \mathrm{~V}$ DC voltage. During $\mathrm{Nb}$ deposition, the

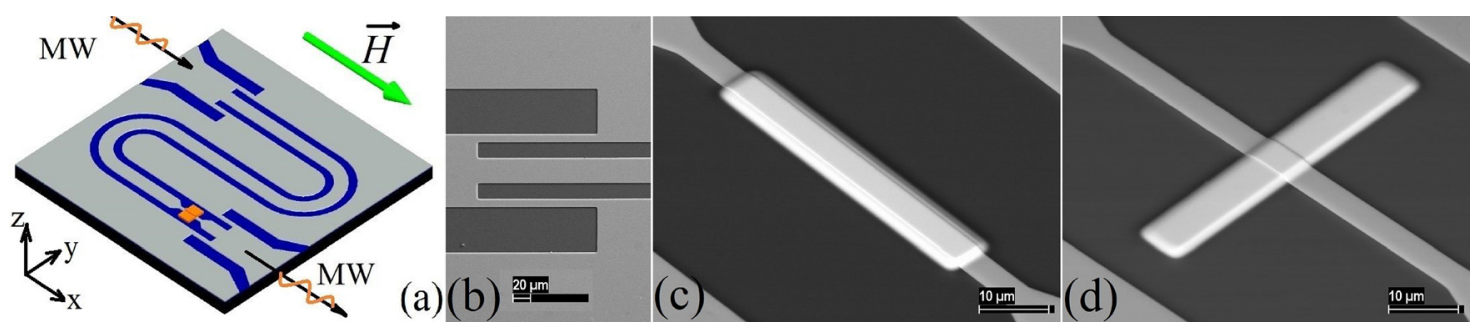

FIG. 1. (a) Schematic illustration of the structure design for probing magnetization dynamics of individual small FM samples (not to scale). A Nb CPW $\lambda / 2$ resonator with inductive coupling (in grey) is patterned on Si substrate (in black). Gaps of the CPW are highlighted in blue. An individual patterned permalloy FM film sample (in orange) is placed onto a constriction in central stripe of the resonator in close vicinity to the inductive coupler. Black and green arrows show, respectively, the direction of propagation of the microwave signal and the direction of the external magnetic field. (b) SEM image of the inductive coupler. (c) SEM image of the FM sample aligned along the transmission line (PA geometry). (d) SEM image of the FM sample aligned across the transmission line (PE geometry). 
argon pressure, $\mathrm{RF}$ power, deposition rate, and $\mathrm{DC}$ voltage were $4 \times 10^{-3} \mathrm{mbar}, 200 \mathrm{~W}, 2.2 \AA / \mathrm{s}$, and $200 \mathrm{~V}$, respectively. Permalloy FM thin film samples with saturation magnetization $M_{s}=8.5 \times 10^{5} \mathrm{~A} / \mathrm{m}$ were deposited onto $\mathrm{Nb}$ resonators using successive magnetron RF-sputtering of Py alloy target and double resist lift-off technique. During Py deposition, the argon pressure, RF power, deposition rate, and DC voltage were $4 \times 10^{-3} \mathrm{mbar}, 200 \mathrm{~W}, 1.5 \AA / \mathrm{s}$, and $450 \mathrm{~V}$, respectively. $\mathrm{AlO}_{x}$ insulating layer was deposited sputtering $\mathrm{Al}$ elemental target in $\mathrm{Ar}+\mathrm{O}_{2}$ atmosphere with $115 \mathrm{sccm}$ of Ar flow and $35 \mathrm{sccm}$ of $\mathrm{O}_{2}$ flow. During $\mathrm{AlO}_{x}$ deposition, $\mathrm{Ar}+\mathrm{O}_{2}$ pressure, $\mathrm{RF}$ power, deposition rate, and DC voltage were $4 \times 10^{-3} \mathrm{mbar}, 200 \mathrm{~W}, 0.6 \AA / \mathrm{s}$, and $510 \mathrm{~V}$, respectively.

Prior to the measurements, the actual fundamental frequency and the resonator quality factor were tested. The frequency of the fundamental resonance obtained is $f_{0}=742 \mathrm{MHz}$; the quality factor $Q$ of the resonance, determined in PE geometry at the fundamental frequency at magnetic field far from the FMR field $H>1500$ Oe, is $Q \sim 10^{4}$. The composition and the saturation magnetization $M_{s}=8.5$ $\times 10^{5} \mathrm{~A} / \mathrm{m}$ of permalloy films were also verified.

\section{RESULTS AND DISCUSSION}

\section{A. FMR response of test samples}

The transmission spectra of the test chip consist of multiple resonances at $f=n \times f_{0}$. Figure 2 shows the stacked normalized spectra $S_{21}(f, H)$ in vicinity of resonance frequencies, where the maximum microwave signal transmission is color-coded with red. For some modes, a resonance splitting can be observed [for example, mode \#3 in Fig. 2(b).] The splitting arises from interaction of PA and PE structures, which were patterned on the same chip, through parasitic substrate modes, and can be avoided by using smaller substrate, by patterning a single structure per substrate. Also, a marginal dependence of the resonance frequency on $H$ is observed, which can be explained by the presence of Abrikosov vortices. ${ }^{54}$

Scanning the magnetic field, the resonant coupling of the FM sample with the resonator is achieved at each resonance mode at field where the FMR frequency matches the resonator frequency $n \times f_{0}$, and the effective absorption of the MW by the FM occurs. The resonant coupling is represented by the substantial drop of the $Q$-factor [on the raw spectra the best seen in Fig. 2(a)]. The dependence of the Qfactor on magnetic field was extracted from experimental data by fitting the resonance curves at each $H$ with a modified model of a through type resonator ${ }^{50}$

$$
\begin{aligned}
S_{21}(f)= & \left(\sum_{k} \frac{A_{k} Q_{k}}{1+2 i Q_{k}\left(f-f_{r, k}\right) / f_{r, k}}+B_{n}(f) \exp \left(i C_{n}(f)\right)\right) \\
& \times \exp \left(i \delta f+\phi_{0}\right),
\end{aligned}
$$

where $k$ is the index of resonant peak [the $k$ indexing is used if two peaks appear, as at mode \#3 in Fig. 2(b)], $Q$ is the loaded quality factor, $A$ is an amplitude scale coefficient, $f_{r}$ is the resonant frequency, $B_{n}(f)$ is the polynomial of degree $n$ representing amplitude of the parallel parasitic transmission through substrate modes, $C_{n}(f)$ is the polynomial representing phase of the parasitic transmission, $\delta$ is the phase slope due to delay in cables, and $\phi_{0}$ is a constant phase shift. A standard complex fitting routine was employed with a parameter set: $Q, f_{r}, A, \delta, \phi_{0}$ and polynomial coefficients $B_{n}(f)$ and $C_{n}(f)$.

As an example, dependencies of the Q-factor on applied magnetic field for several selected resonant modes are demonstrated in Fig. 2(c), where minimums of the $Q(H)$ at FMR are clearly visible and are indicated with arrows. We should note that the line-shape of the $Q(H)$ at the FMR is wide, nonlorentzian, and does not indicate the Gilbert line-width of magnetic resonance. ${ }^{15}$ As will be discussed further, this lineshape is distorted by an overlap with magnetostatic standing wave resonances, as well as due to demagnetization of FM sample at low magnetic fields [flat-like bottom of the dip of black solid line in Fig. 2(c)]. The line-shape of the $Q(H)$ should be close to the line-shape of the FMR for FM samples of appropriate size and with smaller Gilbert damping. Also, additional dependence $Q(H)$ is superimposed on magnetic resonance curve by superconducting vortices. ${ }^{54}$

Taking the position of the minimum of Q-factor for each resonant mode $\left(f=n \times f_{0}, H\right)$, we can restore the dependence of the FMR frequency on applied magnetic field $f_{r}(H)$.
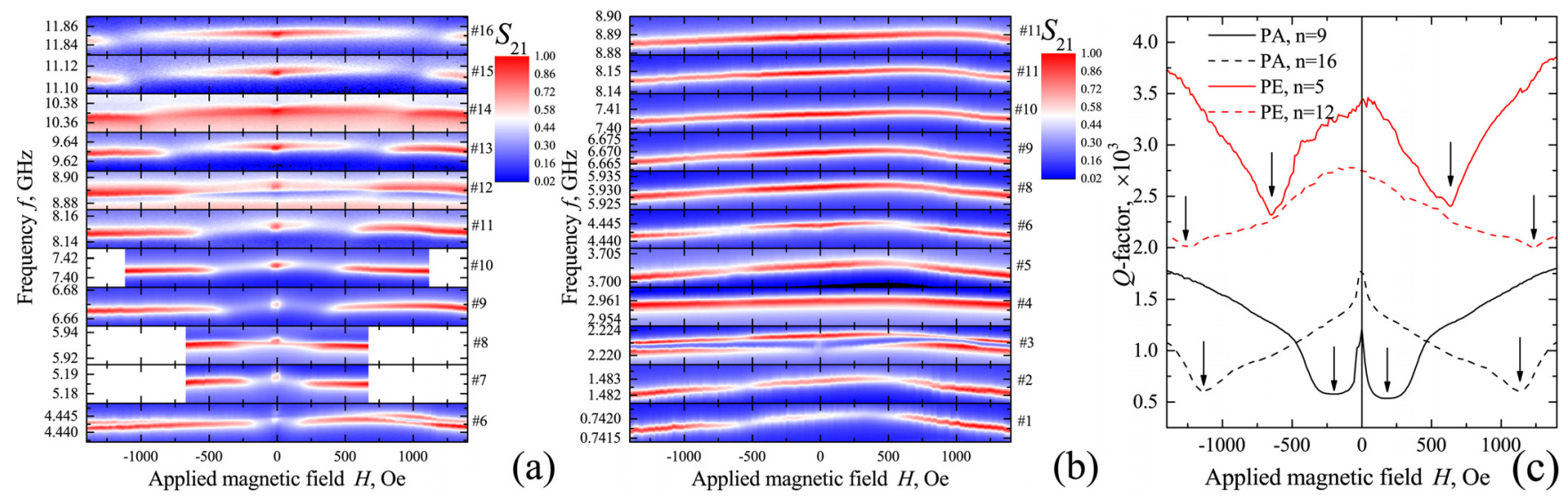

FIG. 2. Normalized transmission spectra $S_{21}$ at resonant modes $n$ (\#) for PA geometry (a) and PE geometry (b). (c) The dependence of the Q-factor on magnetic field for several selected resonant modes $n$. The minimums of the Q-factor are indicated with arrows. 
Dotted data in Fig. 3 show restored $f_{r}(H)$ dependencies. Presuming absence of internal magnetic anisotropies, $f_{r}(H)$ can be fitted using the original Kittel formula for a finite sample

$$
\left(\frac{2 \pi f_{r}}{\mu_{0} \gamma}\right)^{2}=\left(H+\left(N_{y}-N_{x}\right) M_{s}\right)\left(H+\left(N_{z}-N_{x}\right) M_{s}\right),
$$

where $\mu_{0}$ is the vacuum permeability, $\gamma=1.856 \times 10^{11} \mathrm{~Hz} / \mathrm{T}$ is the gyromagnetic ratio, $N_{x}$ is the in-plane demagnetizing factor along the applied magnetic field, $N_{y}$ is the in-plane demagnetizing factor across the applied magnetic field, and $N_{z}$ is the out-of-plane demagnetizing factor.

In general, demagnetizing factor $N$ denotes the average magnetostatic stray self-field $H_{d}$ induced by the saturated ferromagnet itself, i.e., by definition, $H_{d}=-N M_{s}$. First, we attempt to fit the experimental data using the conventional, so-called magnetometric, volume-averaged set of demagnetizing factors. ${ }^{55} \mathrm{FM}$ dimensions yield $N_{x} \approx 0.0129$, $N_{y} \approx 0.0977$, and $N_{z} \approx 0.8894$ for the PA geometry and $N_{x} \approx 0.0977$ and $N_{y} \approx 0.0129$ for the PE geometry. Dependencies $f_{r}(H)$ calculated with magnetometric demagnetizing factors are plotted in Fig. 3 with dotted lines and show a total misfit with the experimental data. This inconsistency has forced us to verify the composition and the saturation magnetization of the FM at the first place.

Now, it should be noted that the functioning demagnetizing factor, i.e., averaged magnetostatic self-field, which determines the FMR frequency or characterizes demagnetization, is not necessary the volume-averaged one. It was shown that for large-area thin film rectangles, the fluxometric demagnetizing factor, i.e., the one where the magnetostatic self-field is averaged over the middle cross-section, is superior to the magnetometric one. ${ }^{32,56} \mathrm{FM}$ dimensions yield the following fluxometric demagnetizing factors $N_{x} \approx 0.0010$, $N_{y} \approx 0.0464$, and $N_{z} \approx 0.8838$ for the PA geometry and

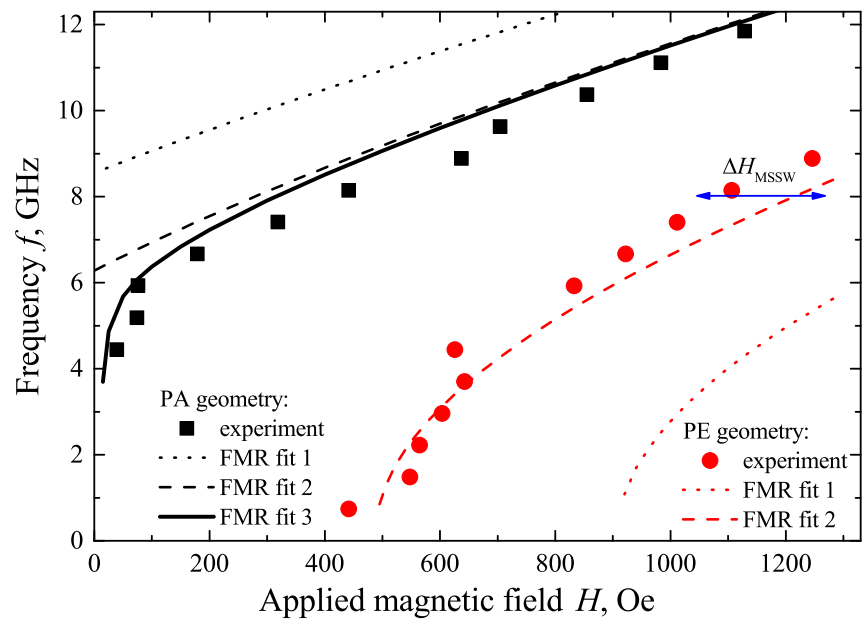

FIG. 3. Dependence of FMR frequency on applied magnetic field. Point data $(f, H)$ correspond to positions of Q-factor minimums $\left(n \times f_{0}, H\right)$ in Fig. 2 . Dotted lines (FMR fit 1) correspond to the fit with Eq. (2) employing magnetometric demagnetizing factor. Dashed lines (FMR fit 2) correspond to the fit with Eq. (2) employing fluxometric demagnetizing factor. Solid line (FMR fit 3) corresponds to the fit with Eq. (2) employing fluxometric demagnetizing factor and micromagnetic simulated relaxing magnetization $M_{x}(H)$ instead of $M_{s}$.
$N_{x} \approx 0.0464$ and $N_{y} \approx 0.0010$ for the PE geometry. Dashed lines in Fig. 3 show $f_{r}(H)$ dependencies calculated with fluxometric demagnetizing factors. The calculated curves match adequately with the experimental data; the mismatch becomes pronounced at $H<200$ Oe for PA geometry and at $H>800$ Oe for PE geometry. Therefore, we confirm the superiority of the fluxometric demagnetizing factor for largearea thin film rectangles. Below, we remove the observed inconsistencies between experimental and calculated fluxometric $f_{r}(H)$ dependencies for PA and PE geometries at low and high fields, respectively.

The Kittel formula [Eq. (2)] considers the ferromagnetic resonance of a completely saturated magnetic moment and obviously disagrees with the observations if demagnetization of the FM sample emerges. Since the demagnetization is expected at low magnetic fields, it can lead to the deviation of the measured $f_{r}(H)$ from the Kittel's one in PA geometry. To explore the effect of possible demagnetization in PA geometry, we simulate the dependence of the averaged magnetization $M_{x}(H)$ on applied magnetic field using micromagnetic simulation. ${ }^{23,24}$ For magnetostatic simulations, we mesh the FM with $40 \times 40 \times 39 \mathrm{~nm}$ cells and, also, account the step geometry of $550 \mathrm{~nm}$ thick and $7 \mu \mathrm{m}$ wide FM sample deposited on top of $100 \mathrm{~nm}$ thick and $5 \mu \mathrm{m}$ wide current carrying conductor [Fig. 1(c)]. Note, since the cell size employed is far above the exchange length in Py, $l_{e} \sim 5 \mathrm{~nm}$ simulations provide incorrect internal magnetic structure. However, the PA sample is much larger than $l_{e}$ in any dimension, and its demagnetization is dominated by competition between the demagnetizing field and the applied field but not the exchange field. Therefore, simulations with the selected cell size provide a sufficiently accurate value of averaged magnetization $M_{x}(H)$ despite the invalid internal magnetic structure.

Figure 4 shows the calculated dependence of the averaged magnetic moment on applied magnetic field $M_{x}(H) / M_{s}$ in PA geometry. Simulations show that the magnetization starts to relax progressively upon decreasing magnetic field at $H<200 \mathrm{Oe}$, reducing the average magnetic moment $M_{x}$. Black solid line in Fig. 3 shows the $f_{r}(H)$ dependence

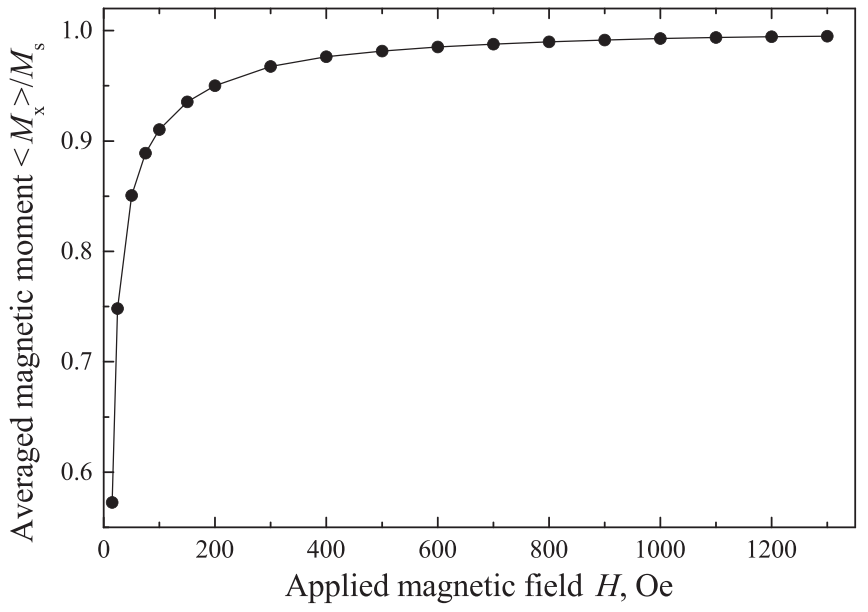

FIG. 4. Simulated dependence of the averaged magnetic moment of the sample in PA geometry on applied magnetic field. Point data are the simulation results and solid line is an eye guide. 


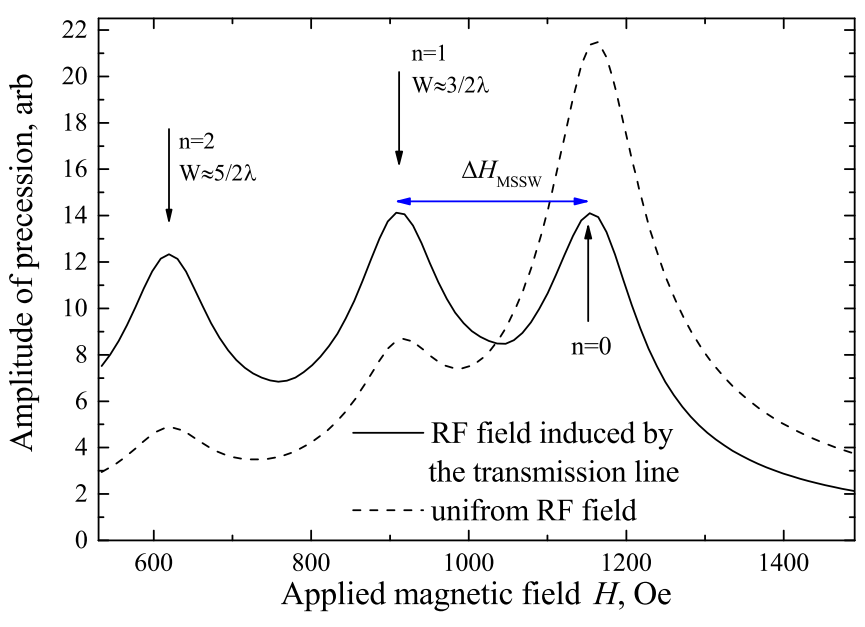

FIG. 5. Simulated dependencies of the volume averaged amplitude of magnetization precession excited by MW magnetic field at $f=8 \mathrm{GHz}$ on applied magnetic field for different spatial distributions of MW excitation field. The maximums are indicated with black arrows.

calculated with fluxometric demagnetizing factors and interpolated $M_{x}(H)$ from Fig. 4 instead of $M_{s}$. The calculated $f_{r}(H)$ curve demonstrates a good match with the experiment on the entire $H$ range, including the low field range. Thus, we confirm the deviation of $f_{r}(H)$ at $H<200$ Oe in PA geometry from the original Kittel formula in Fig. 3 as an indication of demagnetization.

In PE geometry, the deviation of the $f_{r}(H)$ from Eq. (2) is progressive upon increasing magnetic field and can hardly be explained by demagnetization. On the other hand, the localized source of MW magnetic fields in a wide FM sample and overall geometry of the PE experiment suggests nonlocality of magnetization precession and the possibility for excitation of magnetostatic surface wave (MSSW). ${ }^{57-61}$ In thin film geometry, the MSSW propagates with the wave vector $\vec{k}$ aligned in-plane and perpendicular to the applied magnetic field, i.e., along the longest dimension of the FM sample in PE geometry. To capture the MSSW activity, we perform dynamic micromagnetic simulations employing a 2 D $1 \times 1000 \times 12$ mesh with $7 \times 0.05 \times 0.055 \mu \mathrm{m}$ cells, following Ref. 59. The mesh accounts the step geometry of the FM sample deposited on top of the current carrying conductor [Fig. 1(d)]. In the numerical experiment, we apply small microwave magnetic field $\vec{H}_{M W}$ of frequency $f=8 \mathrm{GHz}$ and derive the dependence of the averaged amplitude of the steady state magnetization precession on applied magnetic field $H$. The field of maximum amplitude corresponds to the maximum energy absorption from MW field source, i.e., to the FMR field.

First, we simulate the MW response by applying spatially uniform $\vec{H}_{M W}$ along y-axis (dashed line in Fig. 5). The MW response consists of the main FMR absorption peak at $H \approx 1150 \mathrm{Oe}(n=0)$, where precession is coherent in the entire sample volume, and two weaker resonance peaks with notations $n=1$ and 2 . The later manifests the MSSW resonance with odd half-wavelength quantization with the sample width $W=50 \mu \mathrm{m}$, i.e., $W \approx 3 \lambda / 2$ for $n=1$ and $W \approx 5 \lambda / 2$ for $n=2$.
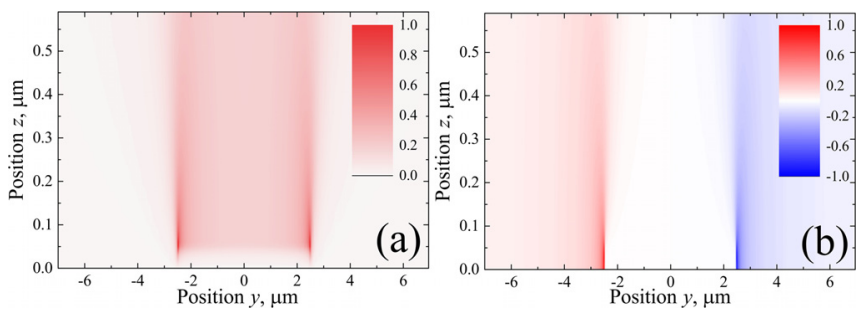

FIG. 6. Calculated distributions $H_{y}(y, z)$ (a) and $H_{z}(y, z)$ (b) of components of the RF magnetic field $\vec{H}_{M W}(y, z)$ induced by the narrow $5 \mu \mathrm{m}$ wide conductor under the FM sample.

Next, we simulate the MW response applying realistic, spatially nonuniform $\vec{H}_{M W}(y, z)$ induced by the transmission line in the FM. The distribution of $\vec{H}_{M W}(y, z)$ was obtained using FEMM software ${ }^{62}$ in a perfect conductor approximation and is shown in Figs. 6(a) and 6(b) for $y-$ and $z-$ components, respectively. The response of the FM excited with the localized MW field is shown in Fig. 5 with solid line and consists of the same three absorption peaks. However, in contrast to the excitation with uniform MW magnetic field, the magnitude of all three absorption peaks is comparable, and the highest absorption is observed for $n=1$ mode at $H \approx 910 \mathrm{Oe}$. The field difference between the FMR and the $n=1$ mode of MSSW resonance $\Delta H_{M S S W} \approx 240 \mathrm{Oe}$ in Fig. 5 (indicated with the blue arrow in Figs. 5 and 3 ) matches reasonably the difference of $130 \mathrm{Oe}$ observed between experimentally defined and Kittel's resonance curves in PE geometry at $8 \mathrm{GHz}$ in Fig. 3. Further discrepancy can be attributed to demagnetization of PE sample. Additionally, we have simulated the MW response in PE geometry by employing spatially nonuniform $\vec{H}_{M W}(y, z)$ given with the Karlqvist equations ${ }^{58}$ and found no qualitative difference with the response in Fig. 5. Thus, the resonant response of the FM sample in PE geometry is represented by the FMR at low fields $H \gtrsim N_{x} M_{S}$ and is shifted to the MSSW resonance with odd half-wavelength quantization at higher fields.

It is worth to mention the distribution of the amplitude of magnetization precession at the MSSW resonance. The distribution of $y$ - and $z$-components of the amplitude at $f=8 \mathrm{GHz}$ and $H=910 \mathrm{Oe}$ (Fig. 7) indicates that the precession amplitude remains finite at both ends of the sample, i.e., the boundary conditions are neither opened nor closed. Uncertain boundary conditions for the magnetostatic standing wave resonance in finite size ferromagnetic objects, as well as nonuniform demagnetization field, obstruct employment of dispersion relations for magnetostatic wave modes. It forces us to use the approximate equality sign instead of the exact equality sign for notation of quantization of the MSSW wavelength $\lambda / 2$ with the FM width $W$ throughout this work. Also, a substantial disparity of the maximum amplitudes in Fig. 7 at opposite sides of the MW carrying stripe, referred commonly as nonreciprocity, ${ }^{59,63}$ is evident. The nonreciprocity can possibly facilitate new approaches for building on-chip devices similar to ferromagnetic circulators and isolators, which can be combined with superconducting microwave circuits, by analogy with nonreciprocal Josephson junction transmitters. ${ }^{64,65}$ 


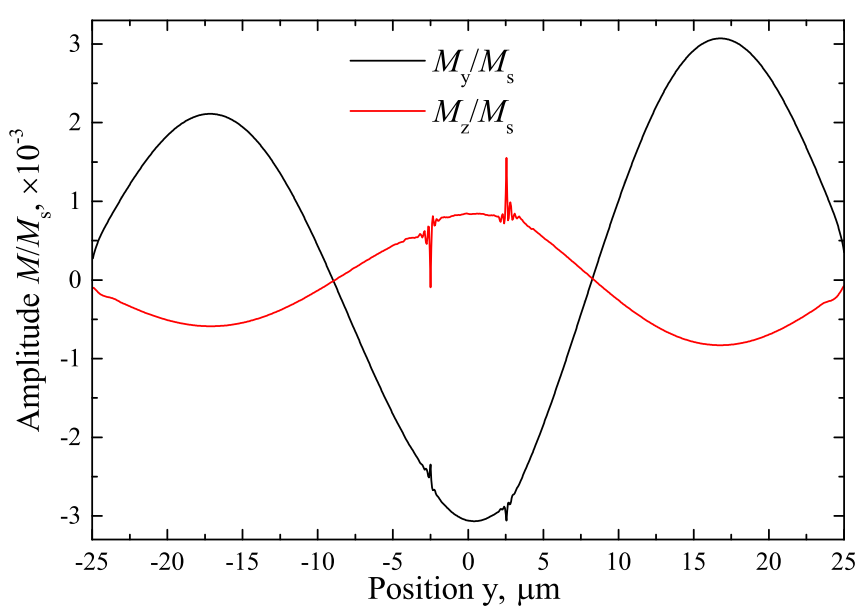

FIG. 7. Simulated distribution of the thickness averaged amplitude of magnetization precession along the y-axis in PE geometry at $f=8 \mathrm{GHz}$ and $H=910$ Oe $(n=1$ mode in Fig. 5$)$.

\section{B. Sensitivity and applicability of superconducting resonator for FMR measurements}

It is important to address the sensitivity and applicability limits of superconducting resonators for FMR measurements. In general, the limiting conditions $(f, \vec{H}, T)$ are attributed to the resistive losses in superconductor. A simple criteria for applicability imply conditions where the internal Q-factor of the resonator $\left(Q_{i}\right)$ remains below the designed coupling Qfactor. At $H=0$, the internal Q-factor can be estimated as $Q_{i}$ $\sim 1 / \alpha_{r} L$, where $\alpha_{r}$ is Clem's microwave quasi-particle attenuation in superconducting coplanar transmission line ${ }^{66}$ associated with Mattis-Bardeen quasi-particle conductivity, ${ }^{67,68}$ and $L=80 \mathrm{~mm}$ is the length of resonator. Figure 8 shows the $\log Q_{i}(f, T)$ colormap calculated using geometrical parameters of the fabricated resonator and the following realistic physical properties of $\mathrm{Nb}$ film: superconducting critical temperature $T_{c}=9.2 \mathrm{~K}$, London penetration depth $\lambda_{L}=80 / \sqrt{\left(1-\left(T / T_{c}\right)^{4}\right)} \mathrm{nm}$, superconducting gap frequency $f_{g}=720 \mathrm{GHz}$, temperature dependence of the superconducting $\operatorname{gap}^{68} \Delta(T)=\Delta(0) \sqrt{\cos \left(\pi / 2\left(T / T_{c}\right)^{2}\right)}$, and normal state conductivity $\sigma_{n}=1.5 \times 10^{7}(\Omega \mathrm{m})^{-1}$. At $T=4 \mathrm{~K}$ used for measurements in this work, $Q_{i}$ approaches $Q=10^{4}$ at $f \sim 40 \mathrm{GHz}$, which is well comparable with a conventional broadband VNA-FMR technique, and at $T=2 \mathrm{~K}$ the internal Q-factor remains above $10^{7}$ even at hundreds of GHz. Therefore, employment of superconducting resonators for FMR measurements does not imply any specific frequency limitations.

Limitations in magnitude and orientation of magnetic field are mainly attributed to attenuation due to flux-creep/ flow resistivity induced by Abrikosov vortices, ${ }^{54,69,70}$ and, fundamentally, by the superconducting upper critical field. At out-of-plane magnetic field, the internal Q-factor of a high-Q transmission line superconducting resonator drops down to $10^{4}$ typically at $\sim 100 \mathrm{mT},{ }^{54}$ which may possess some limitations for FMR measurements at out-of-plane magnetic field. A range of out-of-plane magnetic fields can be extended by considering efficient pinning of vortices. At

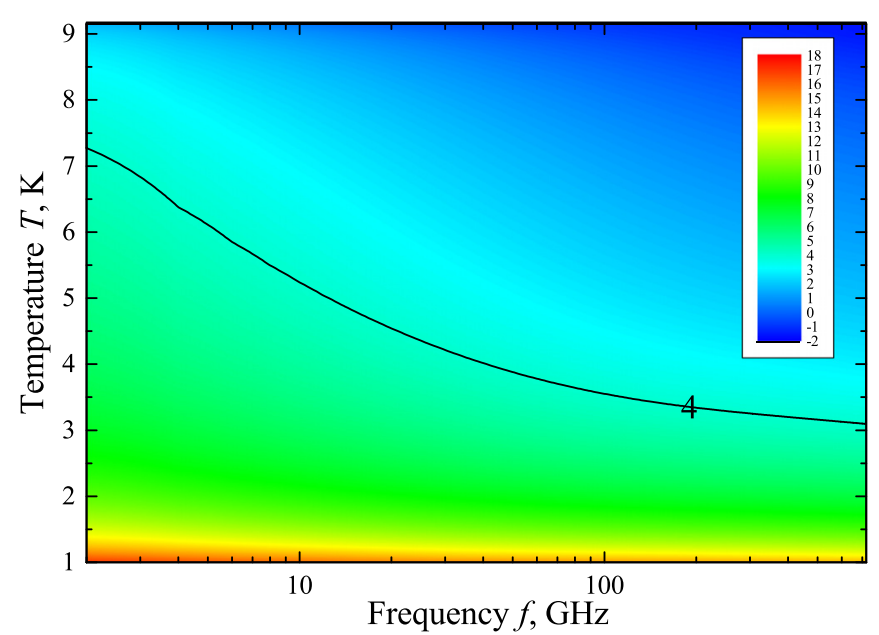

FIG. 8. Colormap dependence of $\log \left(Q_{i}\right)$ on temperature and operation frequency. Solid line indicates $Q=10^{4}$ isoline.

perfectly aligned in-plane magnetic field, the internal Qfactor is not affected by magnetic field at $H$ up to $\sim 300 \mathrm{mT} .{ }^{51}$ If a nucleation of Abrikosov vortices in superconducting resonant structure can be avoided, the Q-factor is unaffected by the magnetic field at $H$ up to several Tesla. ${ }^{70}$

An accurate determination of sensitivity limits requires additional studies with variable sample size. However, the sensitivity of the approach can be illustrated using the results of PE experiment at low resonance modes (\#1 and \#2) [Figs. 1(a), 1(d), and 2(b)] as follows. Micromagnetic simulations show that at resonance modes \#1 and \#2 in PE geometry, the precession of magnetic moment, which causes the drop of $S_{21}$, occurs just above the resonator [see Fig. 1(d)], i.e., on the width of approximately $5 \mu \mathrm{m}$. Thus, the investigated sample of $50 \mu \mathrm{m}$ width in PE geometry provides the same FMR absorption as $5 \mu \mathrm{m}$ size rectangle. Also, the AC excitation field is nonuniform along $z$-axis (Fig. 6), the coupling to the resonator is much stronger for magnetic spins in vicinity, and, therefore, a thinner sample of $100 \mathrm{~nm}$ thick will provide a similar well measurable drop of $S_{21}$ at the resonance in PE geometry, as the original $550 \mathrm{~nm}$ thick one. Therefore, a measurement of a $7 \times 5 \times 0.1 \mu \mathrm{m}^{3}$ sample can be performed with the microwave design employed instead of a $7 \times 50 \times 0.55 \mu \mathrm{m}^{3}$, providing the same drop of Q-factor. Next, the coupling can be enhanced by using thinner insulating layer or narrower central line. In particular, narrower samples can be measured with higher coupling strength using narrower constriction in Fig. 1 (the coupling is proportional to the average density of AC and will be enhanced by factor of 5 when the constriction is narrowed further from $5 \mu \mathrm{m}$ to $1 \mu \mathrm{m})$. This scales a measurable size of the FM down to $7 \times 1 \times 0.1 \mu \mathrm{m}^{3}$, by a factor of 275 smaller than the original size. Therefore, the amount of measurable magnetic moments drops from $1.8 \times 10^{13}$ to $6 \times 10^{10}$. At last, the sensitivity of the proposed technique correlates with the Q-factor of the resonator. We have used the resonator with $Q \sim 10^{4}$. Nowadays, superconducting resonators with $Q \sim 10^{5}-10^{6}$ are widely used for bolometers and superconducting qubits measurements. Therefore, an appropriate microwave design of the coupling element [Fig. 1(b)] would increase the Q- 
factor by an order of magnitude and, correspondingly, would reduce the amount of measurable magnetic moments by another order.

\section{CONCLUSION}

Summarizing, in this work, we have proposed and demonstrated a sensitive, flexible approach for probing magnetization dynamics of individual micro-scaled ferromagnets. The approach is based on coupling small FM sample with high-Q multi-mode transmission line superconducting resonator and identification of the ferromagnetic resonance by measuring changes of its resonant line profile while sweeping bias magnetic field. The sensitivity of the technique can be enhanced further drastically by employing resonators with higher quality factor, using a thinner insulating layer or narrower central line.

Additionally, studying the MW response of microscaled Py thin film rectangular samples, we have confirmed the superiority of the fluxometric demagnetizing factor for large-area thin film rectangles over the commonly accepted magnetometric one. We have observed deviations between the experimental and Kittel's FMR curves, which we attribute to the demagnetization of the FM sample and to magnetostatic surface wave resonance.

\section{ACKNOWLEDGMENTS}

I.A.G., N.N.A., A.S.A., V.V.R., and A.V.U. acknowledge the Ministry of Education and Science of the Russian Federation (Research Project Nos. K2-2014-025 and K2-2016051 in the framework of the Increase Competitiveness Program of NUST "MISiS") for support in microwave measurements. V.S.S., I.A.G., and A.A.G. acknowledge the joint Russian-

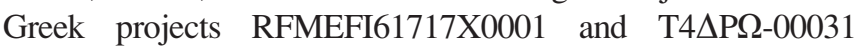
"Experimental and theoretical studies of physical properties of low-dimensional quantum nanoelectronic systems" for support in samples fabrication. I.A.G. acknowledges partial support from the Russian Foundation for Basic Research (RFBR) (Project No. 16-32-00309). V.S.S. and V.V.R. acknowledge partial support from the Program of Competitive Growth of Kazan Federal University.

${ }^{1}$ G. A. Prinz, Science 282, 1660 (1998).

${ }^{2}$ S. A. Wolf, D. D. Awschalom, R. A. Buhrman, J. M. Daughton, S. von Molnár, M. L. Roukes, A. Y. Chtchelkanova, and D. M. Treger, Science 294, 1488 (2001).

${ }^{3}$ C. A. Ross, S. Haratani, F. J. Castaño, Y. Hao, M. Hwang, M. Shima, J. Y. Cheng, B. Vögeli, M. Farhoud, M. Walsh et al., J. Appl. Phys. 91, 6848 (2002).

${ }^{4}$ O. Rousseau, B. Rana, R. Anami, M. Yamada, K. Miura, S. Ogawa, and Y. Otani, Sci. Rep. 5, 9873 (2015).

${ }^{5}$ E. C. Gingrich, B. M. Niedzielski, J. A. Glick, Y. Wang, D. L. Miller, R. Loloee, W. P. Pratt, Jr., and N. O. Birge, Nat. Phys. 12, 564 (2016).

${ }^{6}$ B. Baek, W. H. Rippard, S. P. Benz, S. E. Russek, and P. D. Dresselhaus, Nat. Commun. 5, 3888 (2014).

${ }^{7}$ V. V. Ryazanov, V. A. Oboznov, A. Y. Rusanov, A. V. Veretennikov, A. A. Golubov, and J. Aarts, Phys. Rev. Lett. 86, 2427 (2001).

${ }^{8}$ L. R. Tagirov, Phys. Rev. Lett. 83, 2058 (1999).

${ }^{9}$ A. K. Feofanov, V. A. Oboznov, V. V. Bol'ginov, J. Lisenfeld, S. Poletto, V. V. Ryazanov, A. N. Rossolenko, M. Khabipov, D. Balashov, A. B. Zorin et al., Nat. Phys. 6, 593 (2010).

${ }^{10}$ H. Hilgenkamp, V. V. Moshchalkov, and P. Kes, Science 302, 1159 (2003).
${ }^{11}$ J. E. Villegas, S. Savel'ev, F. Nori, E. M. Gonzalez, J. V. Anguita, R. García, and J. L. Vicent, Science 302, 1188 (2003).

${ }^{12}$ D. P. de Lara, M. Erekhinsky, E. M. Gonzalez, Y. J. Rosen, I. K. Schuller, and J. L. Vicent, Phys. Rev. B 83, 174507 (2011).

${ }^{13}$ Ferromagnetic Resonance - Theory and Applications, edited by O. Yalcin (InTech, 2013).

${ }^{14}$ I. Neudecker, G. Woltersdorf, B. Heinrich, T. Okuno, G. Gubbiotti, and C. Back, J. Magn. Magn. Mater. 307, 148 (2006).

${ }^{15}$ S. S. Kalarickal, P. Krivosik, M. Wu, C. E. Patton, M. L. Schneider, P. Kabos, T. J. Silva, and J. P. Nibarger, J. Appl. Phys. 99, 093909 (2006).

${ }^{16}$ C. Kittel, Phys. Rev 73, 155 (1948).

${ }^{17}$ N. Àlvarez, G. Alejandro, J. Gòmeza, E. Goovaerts, and A. Butera, J. Phys. D: Appl. Phys. 46, 505001 (2013).

${ }^{18}$ Y.-C. Chen, D.-S. Hung, Y.-D. Yao, S.-F. Lee, H.-P. Ji, and C. Yu, J. Appl. Phys. 101, 09C104 (2007).

${ }^{19}$ Y. V. Khivintsev, L. Reisman, J. Lovejoy, R. Adam, C. M. Schneider, R. E. Camley, and Z. J. Celinski, J. Appl. Phys. 108, 023907 (2010).

${ }^{20}$ J. Wei, Z. Zhu, C. Song, H. Feng, P. Jing, X. Wang, Q. Liu, and J. Wang, J. Phys. D: Appl. Phys. 49, 265002 (2016)

${ }^{21}$ H. Puszkarski and P. Tomczak, Sci. Rep. 4, 6135 (2014).

${ }^{22}$ H. Puszkarski, P. Tomczak, and H. T. Diep, Phys. Rev. B 94, 195303 (2016).

${ }^{23}$ M. Donahue and D. Porter, "OOMMF user's guide, version 1.0," Interagency Report No. NISTIR 6376, National Institute of Standards and Technology, Gaithersburg, MD, 1999.

${ }^{24}$ J. E. Miltat and M. J. Donahue, "Numerical micromagnetics: Finite difference methods," in Handbook of Magnetism and Advanced Magnetic Materials (John Wiley \& Sons, Ltd., 2007), Vol. 2.

${ }^{25}$ V. Novosad, F. Y. Fradin, P. E. Roy, K. S. Buchanan, K. Y. Guslienko, and S. D. Bader, Phys. Rev. B 72, 024455 (2005).

${ }^{26}$ A. A. Awad, K. Y. Guslienko, J. F. Sierra, G. N. Kakazei, V. Metlushko, and F. G. Aliev, Appl. Phys. Lett. 96, 012503 (2010).

${ }^{27}$ G. N. Kakazei, G. R. Aranda, S. A. Bunyaev, V. O. Golub, E. V. Tartakovskaya, A. V. Chumak, A. A. Serga, B. Hillebrands, and K. Y. Guslienko, Phys. Rev. B 86, 054419 (2012).

${ }^{28}$ N. Ross, M. Kostylev, and R. L. Stamps, J. Appl. Phys. 116, 113909 (2014).

${ }^{29}$ K. Y. Guslienko, G. N. Kakazei, J. Ding, X. M. Liu, and A. O. Adeyeye, Sci. Rep. 5, 13881 (2015).

${ }^{30}$ J. Ding, P. Lapa, S. Jain, T. Khaire, S. Lendinez, W. Zhang, M. B. Jungfleisch, C. M. Posada, V. G. Yefremenko, J. E. Pearson et al., Sci. Rep. 6, 25196 (2016).

${ }^{31}$ E. V. Skorohodova, R. V. Gorev, R. R. Yakubov, E. S. Demidov, Y. V. Khivintsev, Y. A. Filimonov, and V. L. Mironov, J. Magn. Magn. Mater. 424, 118 (2017).

${ }^{32}$ Y. Li, Y. Lu, and W. E. Bailey, J. Appl. Phys. 113, 17 B506 (2013).

${ }^{33}$ I. A. Golovchanskiy, V. V. Bolginov, N. N. Abramov, V. S. Stolyarov, A. B. Hamida, V. I. Chichkov, D. Roditchev, and V. V. Ryazanov, J. Appl. Phys. 120, 163902 (2016).

${ }^{34}$ C. S. Chang, M. Kostylev, A. O. Adeyeye, M. Bailleul, and S. Samarin, EPL 96, 57007 (2011).

${ }^{35}$ K. Vogt, H. Schultheiss, S. J. Hermsdoerfer, P. Pirro, A. A. Serga, and B. Hillebrands, Appl. Phys. Lett. 95, 182508 (2009).

${ }^{36}$ T. Sebastian, Y. Kawada, B. Obry, T. Brächer, P. Pirro, D. A. Bozhko, A. A. Serga, H. Naganuma, M. Oogane, Y. Ando et al., J. Phys. D: Appl. Phys. 48, 164015 (2015).

${ }^{37}$ K. Perzlmaier, M. Buess, C. H. Back, V. E. Demidov, B. Hillebrands, and S. O. Demokritov, Phys. Rev. Lett. 94, 057202 (2005).

${ }^{38}$ H. T. Nembach, J. M. Shaw, C. T. Boone, and T. J. Silva, Phys. Rev. Lett. 110, 117201 (2013).

${ }^{39}$ T. Mewes, J. Kim, D. V. Pelekhov, G. N. Kakazei, P. E. Wigen, S. Batra, and P. C. Hammel, Phys. Rev. B 74, 144424 (2006).

${ }^{40}$ O. Klein, G. de Loubens, V. V. Naletov, F. Boust, T. Guillet, H. Hurdequint, A. Leksikov, A. N. Slavin, V. S. Tiberkevich, and N. Vukadinovic, Phys. Rev. B 78, 144410 (2008).

${ }^{41}$ H.-J. Chia, F. Guo, L. M. Belova, and R. D. McMichael, Phys. Rev. Lett. 108, 087206 (2012).

${ }^{42}$ S. Tamaru, K. Yakushiji, A. Fukushima, S. Yuasa, and H. Kubota, IEEE Magn. Lett. 5, 3700304 (2014).

${ }^{43}$ S. Zhang, S. A. Oliver, N. E. Israeloff, and C. Vittoria, Appl. Phys. Lett. 70, 2756 (1997).

${ }^{44}$ A. Banholzer, R. Narkowicz, C. Hassel, R. Meckenstock, S. Stienen, O. Posth, D. Suter, M. Farle, and J. Lindner, Nanotechnology 22, 295713 (2011). 
${ }^{45}$ C. Schoeppner, K. Wagner, S. Stienen, R. Meckenstock, M. Farle, R. Narkowicz, D. Suter, and J. Lindner, J. Appl. Phys. 116, 033913 (2014).

${ }^{46}$ S. E. Barnes, M. Aprili, I. Petkovic, and S. Maekawa, Supercond. Sci. Technol. 24, 024020 (2011).

${ }^{47}$ S. Mai, E. Kandelaki, A. F. Volkov, and K. B. Efetov, Phys. Rev. B 84, 144519 (2011).

${ }^{48}$ I. A. Golovchanskiy, N. N. Abramov, V. S. Stolyarov, O. V. Emelyanova, A. A. Golubov, A. V. Ustinov, and V. V. Ryazanov, Supercond. Sci. Technol. 30, 054005 (2017).

${ }^{49}$ M. Göppl, A. Fragner, M. Baur, R. Bianchetti, S. Filipp, J. M. Fink, P. J. Leek, G. Puebla, L. Steffen, and A. Wallraff, J. Appl. Phys. 104, 113904 (2008).

${ }^{50}$ D. M. Pozar, Microwave Engineering, 4th ed. (John Wiley \& Sons, Inc., 2011).

${ }^{51}$ H. Huebl, C. W. Zollitsch, J. Lotze, F. Hocke, M. Greifenstein, A. Marx, R. Gross, and S. T. B. Goennenwein, Phys. Rev. Lett. 111, 127003 (2013).

${ }^{52}$ Y. Tabuchi, S. Ishino, T. Ishikawa, R. Yamazaki, K. Usami, and Y. Nakamura, Phys. Rev. Lett. 113, 083603 (2014).

${ }^{53}$ X. Zhang, C.-L. Zou, L. Jiang, and H. X. Tang, Phys. Rev. Lett. 113, 156401 (2014).

${ }^{54}$ C. Song, T. W. Heitmann, M. P. DeFeo, K. Yu, R. McDermott, M. Neeley, J. M. Martinis, and B. L. T. Plourde, Phys. Rev. B 79, 174512 (2009).

${ }^{55}$ A. Aharoni, J. Appl. Phys. 83, 3432 (1998).

${ }^{56}$ A. Aharoni, L. Pust, and M. Kief, J. Appl. Phys. 87, 6564 (2000).
${ }^{57}$ S. O. Demokritov, B. Hillebrands, and A. N. Slavin, Phys. Rep. 348, 441 (2001).

${ }^{58}$ J. H. Kwon, S. S. Mukherjee, P. Deorani, M. Hayashi, and H. Yang, Appl. Phys. A 111, 369 (2013).

${ }^{59}$ P. Deorani, J. H. Kwon, and H. Yang, Curr. Appl. Phys. 14, S129 (2014).

${ }^{60}$ A. A. Serga, A. V. Chumak, and B. Hillebrands, J. Phys. D: Appl. Phys. 43, 264002 (2010).

${ }^{61}$ P. Wessels, A. Vogel, J.-N. Tödt, M. Wieland, G. Meier, and M. Drescher, Sci. Rep. 6, 22117 (2016)

${ }^{62} \mathrm{See}$ http://www.femm.info/wiki/HomePage for FEMM software details.

${ }^{63}$ J. H. Kwon, J. Yoon, P. Deorani, J. M. Lee, J. Sinha, K.-J. Lee, M. Hayashi, and H. Yang, Sci. Adv. 2, e1501892 (2016).

${ }^{64}$ K. G. Fedorov, S. V. Shitov, H. Rotzinger, and A. V. Ustinov, Phys. Rev. B 85, 184512 (2012).

${ }^{65}$ A. L. Pankratov, K. G. Fedorov, M. Salerno, S. V. Shitov, and A. V. Ustinov, Phys. Rev. B 92, 104501 (2015).

${ }^{66}$ J. R. Clem, J. Appl. Phys. 113, 013910 (2013).

${ }^{67}$ D. C. Mattis and J. Bardeen, Phys. Rev. 111, 412 (1958).

${ }^{68}$ C. P. McClay, S. Soares, and P. S. Weitzman, Superconducting Microwave Transmission Lines (John Wiley \& Sons, Inc., 1991).

${ }^{69}$ D. Janjusevic, M. S. Grbic, M. Pozek, A. Dulcic, D. Paar, B. Nebendahl, and T. Wagner, Phys. Rev. B 74, 104501 (2006).

${ }^{70}$ N. Samkharadze, A. Bruno, P. Scarlino, G. Zheng, D. P. DiVincenzo, L. DiCarlo, and L. M. K. Vandersypen, Phys. Rev. Appl. 5, 044004 (2016). 